\title{
Approach to Engineering the Temperature Sensing E-textile: A Lightweight Thermistor as an Active Sensing Element
}

\author{
Victor Lebedev ${ }^{1}$, Elena Laukhina ${ }^{1,2(\bowtie)}$, Vladimir Laukhin ${ }^{1,2,3}$, \\ Andrey Somov ${ }^{4}$, Alexander Baranov ${ }^{5}$, Concepcio Rovira ${ }^{1,2}$, \\ and Jaume Veciana ${ }^{1,2}$ \\ ${ }^{1}$ Institut de Ciencia de Materials de Barcelona (ICMAB-CSIC), Campus UAB, \\ 08193 Bellaterra, Spain \\ laukhina@icmab.es \\ ${ }^{2}$ CIBER de Bioingeniería, Biomateriales y Nanomedicina (CIBER-BBN), \\ Madrid, Spain \\ ${ }^{3}$ Institució Catalana de Recerca i Estudis Avançats (ICREA), Barcelona, Spain \\ ${ }^{4}$ CREATE-NET, Trento, Italy \\ 5 'MATI'-Russian State Technological University, Moscow, Russia
}

\begin{abstract}
In this paper, we describe an approach to fabricating conductive textiles with temperature sensing capability. The key point of our approach is in combining electronic properties of a molecular organic semiconductor with clothing. A polycarbonate film covered with organic molecular semiconductor was used as the temperature measurement element. To minimize the electrical response of the developed bi layer thermistor to deformations, the thermistor was attached to a rigid film-like platform specifically fabricated in the textile by its local melting. Our study shows that the developed platform enables engineering of the conductive fabric the electrical resistance of which exclusively responded to temperature changes. Such e-textiles may be easily prepared using a simple fabrication procedure and, therefore, they are compatible with conductive sensing fabrics prepared by printing techniques. The developed organic thermistor, being cheap, lightweight and biocompatible, is highly attractive for applications in wearable biomedical technology.
\end{abstract}

Keywords: E-textile $\cdot$ Bi layer sensing systems $\cdot$ Thermistors $\cdot$ Sensors for wearable technologies

\section{Introduction}

Temperature, deformation and pressure are among the crucial parameters to be measured in a number of sensing applications $[1,2]$ using the state-of-the-art sensing technologies, e.g. wireless sensor network (WSN) [3], wearable technology [4], e-textile [9]. Indeed, healthcare, environment control, biomedical applications call for low cost, lightweight thermistors and piezoresitors which are able to accurately measure temperature and pressure changes [5-10]. 
With the emergence of above mentioned monitoring techniques the sensing technology on plastic substrates has become of especial research interest. It is expected that this technology will build up sensors introduced to new settings by significantly reducing their production cost and by adding new functionalities [5].

As the world population is ageing [11], the need in controlling the personal health status at and out-of home has been constantly increased. In this regard, there is also a particular interest in integrating lightweight conductive sensing materials in human wearable interfaces, such as fabrics, since wearable electronics could offer personalized healthcare, security and comfort $[6,8,9]$. These fabrics are able to sense and react to environmental conditions.

In this work, we present and discuss a new fabricating approach to processing the BL film-like thermistor into polyester textile which enables engineering of e-textiles being capable of controlling very small temperature changes with accuracy of 0.005 degree.

The paper is organized as follows: Sect. 2 will discuss related work in the field. Section 3 presents our approach and experimental results. Finally, we discuss our future work and provide conclusions in Sects. 4 and 5, respectively.

\section{Related Work}

The fabric-based sensing is a large field of research in the biomedicine. As M. Stoppa and A. Chiolerio have pointed in their recent review on e-textiles [9] the fabric sensors may be used for electrocardiogram (ECG) [9], electromyography (EMG) [12], and electroencephalography (EEG) [13, 14] sensing. Modern technologies developing for the sport, military and aerospace also call for sensing e-textiles. Many sensors and actuators have been developed, which are imparted to the fabric during finishing [5-8]. As measured elements they mainly utilize thin metallic wires being embedded in a fabric [9]. Electrically conductive fibers can also be prepared by coating the fibers with metals, galvanic substances or metallic salts. To fabricate electrically conductive textiles, the surface of a textile may also be coated with a thin layer of either metal or semiconductor. Developing metalized plastics [15] and conductive polymers [16] opens new opportunities for engineering e-textiles: coating textiles with conductive sensing plastics. It should be noted, however, that the major problems of metalized plastics are (i) poor adhesion of conventional metals to polymers that are chemical inertness [17, 18], (ii) significant difference in the Young's modules between soft plastics and rigid inorganic metals. These disadvantages result in low binding between the metallic and plastic layers $[17,18]$. On the other hand, conducting polymers are not stable materials: their electronic properties are often unstable towards atmospheric moisture [19]. There are also others barriers to their applications, such as the high manufacturing costs, material inconsistencies, and poor solubility in solvents. In this context, one of the aims of our research was developing of the stable, flexible, composite materials that may be used as low cost, lightweight, conductive sensing components for their applications in wearable biomedical technology. We have recently 


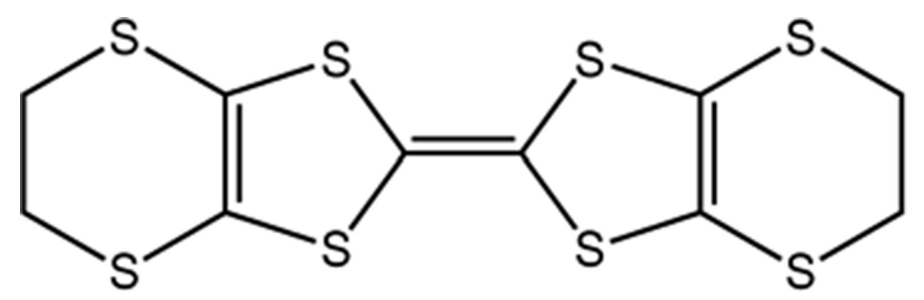

Fig. 1. Skeletal formula of bis(ethylenedithio)tetrathiafulvalen (BEDT-TTF)

developed an original approach to engineering such sensing materials which lies in preparing bi layer (BL) films polycarbonate/(001) oriented organic molecular conductor (BEDT-TTF $)_{2} \mathrm{X}$, were BEDT-TTF = bis(ethylenedithio)tetrathiafulvalen (Fig. 1) and " $\mathrm{X}$ " is trihalide ions [20, 21].

These conductors are charge transfer salts with a $1 / 2$ filled conducting band; they have very deformable layered crystal structures with strong electron-phonon coupling and due to this they demonstrate unique electronic properties that may be exploited in numerous sensing applications [22-24]. It has already been demonstrated that such BL films show a high piezo-resistive effect and could be successfully embedded in textiles as either strain or pressure sensors [25]. This result prompted us to apply the BEDT-TTF-based conductors to engineering conductive fabric whose resistance will be able to respond to small temperature changes.

\section{Results and Discussion}

The development of e-textiles at its first phase is reduced to engineering of sensing materials with electrical detection principle. It should be noted that modern wearable technologies are ideally suited for the electrical detection. In the second phase, it is to be considered how the developed sensing materials may be processed into textiles. In this context, we have developed a BL thermistor: polycarbonate/ $\alpha$-(BEDTTTF $)_{2} \mathrm{I}_{\mathrm{x}} \mathrm{Br}_{3-\mathrm{x}}$, were $\alpha^{\prime}$-(BEDT-TTF $)_{2} \mathrm{I}_{\mathrm{x}} \mathrm{Br}_{3-\mathrm{x}}$ is organic molecular semiconductor the electrical resistance of which is highly sensitive to temperature. In the body temperature range its temperature coefficient of resistance (TCR) is $-1.4 \% / \mathrm{deg}$. This value is 4 times greater than that reported for the platinum thermometer $\left(\mathrm{TCRPt}_{111}=0.3\right)$ [26]. We have recently pointed out [27] that this thermistor may be processed into textile using an impregnation procedure as it is shown in Figs. 2 and 3. However, our latest microscopic study revealed that this attaching approach provoked the formation of some cracks on the conductive sensing layer of the BL thermistor: polycarbonate/ $\alpha$ (BEDT-TTF $)_{2} \mathrm{I}_{\mathrm{x}} \mathrm{Br}_{3-\mathrm{x}}$ (Fig. 4).

The SEM data stimulated us to look for another approach to the second phase. The experimental details of the developed fabricating method, as well as its advantages, will be presented in next sections. 


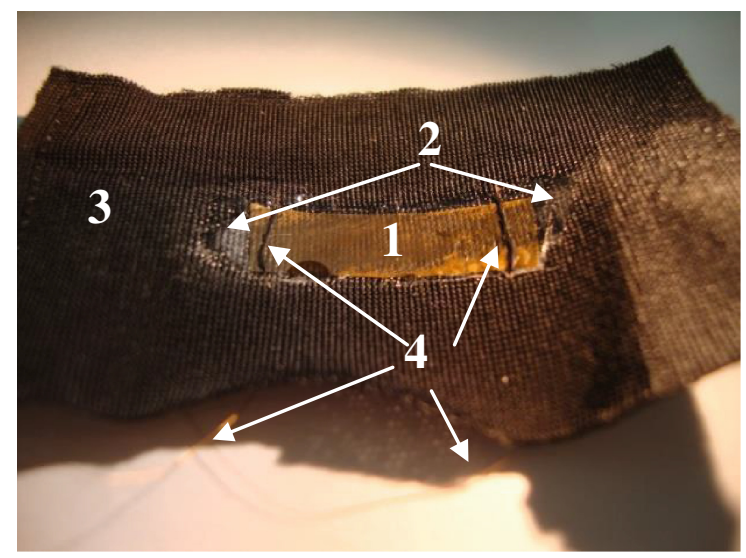

Fig. 2. Photo image of the fabricated e-textile: BL thermistor (1) is attached to the rigid impregnated part (2) of the polyester fabric (3); 4-electrical connections

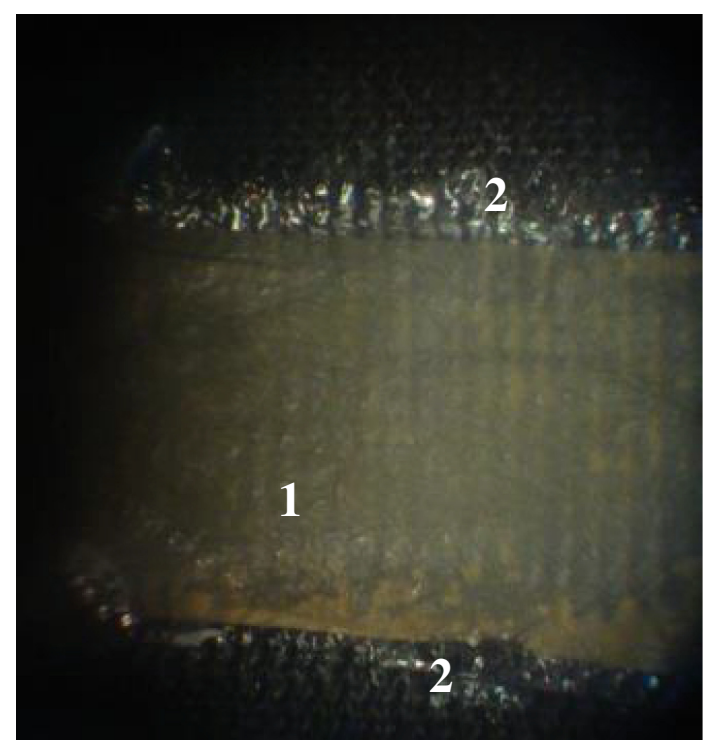

Fig. 3. Photo image of the BL thermistor (1) that was attached to the rigid impregnated part of the polyester fabric (2)

\subsection{Preparation and Characterization of the Flexible Lightweight BL Thermistor: Polycarbonate/ $\alpha$ '-(BEDT-TTF $)_{2} \mathbf{I}_{\mathbf{x}} \mathbf{B r}_{3-\mathbf{x}}$}

In line with the reported synthetic procedure the BL thermistor was fabricated as follows: first, a $25 \mu \mathrm{m}$ thick polycarbonate (PC) film which contains a $2 \mathrm{wt} \%$ of BEDT-TTF was prepared. To do this, the film was cast on a glass support at $130{ }^{\circ} \mathrm{C}$ from a 1,2-dichlorobenzene solution of polycarbonate and BEDT-TTF. Second, to 


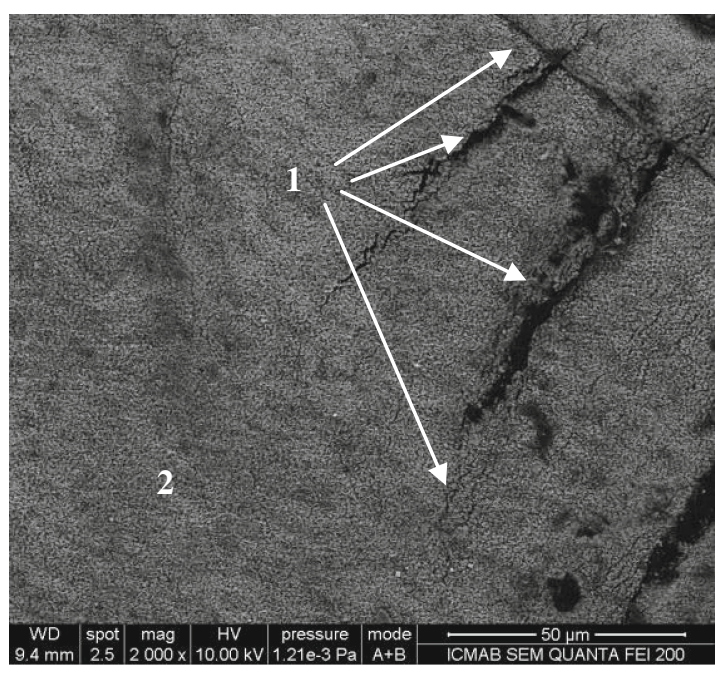

Fig. 4. The Scanning Electron Microscopy (SEM) image of the cracks (1) developed on the conductive sensing layer of the BL thermistor (2) attached to the rigid impregnated part of the polyester fabric

cover the film with the (001) oriented layer of $\alpha^{\prime}-(\mathrm{BEDT}-\mathrm{TTF})_{2} \mathrm{I}_{\mathrm{x}} \mathrm{Br}_{3-\mathrm{x}}$, we exposed the film surface to the vapors of a dichloromethane solution of IBr. The surface of the film easily swells under this treatment, which facilitates the migration of BEDT-TTF molecules from the bulk of the film to the swollen film surface where they are oxidized by IBr. This redox process induces the rapid nucleation of the (BEDT-TTF) ${ }_{2} \mathrm{I}_{\mathrm{x}} \mathrm{Br}_{\mathrm{x}-3}$ conductor with a consequent formation of the conductive polycrystalline covering layer. The resulting surface-modified film was fully characterized using Scanning Electron Microscopy (SEM) and X-ray diffraction techniques; its $R(T)$ dependence in the range of the human body temperatures was also investigated.

Figure 5 demonstrates that the sensing layer of the BL thermistor polycarbonate/ $\alpha$ (BEDT-TTF $)_{2} \mathrm{I}_{\mathrm{x}} \mathrm{Br}_{3-\mathrm{x}}$ consists of submicro plate-like crystallites the crystal plans of which are oriented in parallel to the BL thermistor plan.

The powder X ray diffraction pattern (Fig. 6) shows only (OOl) reflections of the $\alpha^{\prime}$ $(\mathrm{BEDT}-\mathrm{TTF})_{2} \mathrm{I}_{\mathrm{x}} \mathrm{Br}_{3-\mathrm{x}}$ semiconductor. Therefore, the sensing layer of the thermistor has (001) orientation; it means that the layer is dominantly formed from $c^{*}$-oriented $\alpha$ $(\mathrm{BEDT}-\mathrm{TTF})_{2} \mathrm{I}_{\mathrm{x}} \mathrm{Br}_{3-\mathrm{x}}$ crystallites. The direct current resistance measurements demonstrate that the value of the room temperature sheet resistance of the developed BL thermistor is $\cong 30 \mathrm{k} \Omega / \mathrm{cm}^{2}$ and its conductance shows the linear temperature dependence in the temperature range from 28 to $50{ }^{\circ} \mathrm{C}$; the sensitivity of the thermistor sheet resistance to temperature was as $250-300 \Omega / \mathrm{cm}^{2}$ per degree.

In conclusion of this section, we would like to note that the above presented SEM and X-ray data, as well as the resistance temperature dependence, are in accordance with the early reported ones [21, 27]. 


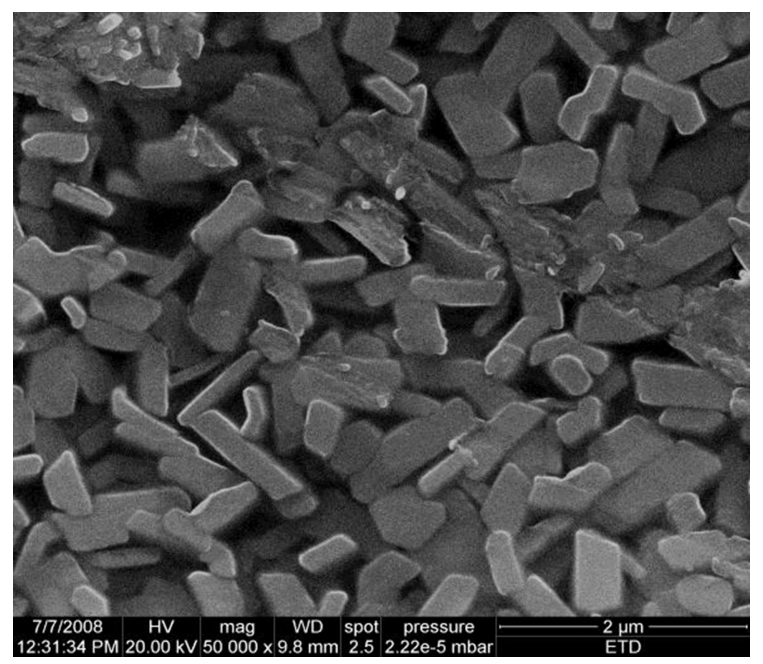

Fig. 5. SEM image of the conductive sensing layer of the BL thermistor: polycarbonate/ $\alpha$ '$(\mathrm{BEDT}-\mathrm{TTF})_{2} \mathrm{I}_{\mathrm{x}} \mathrm{Br}_{3-\mathrm{x}}$

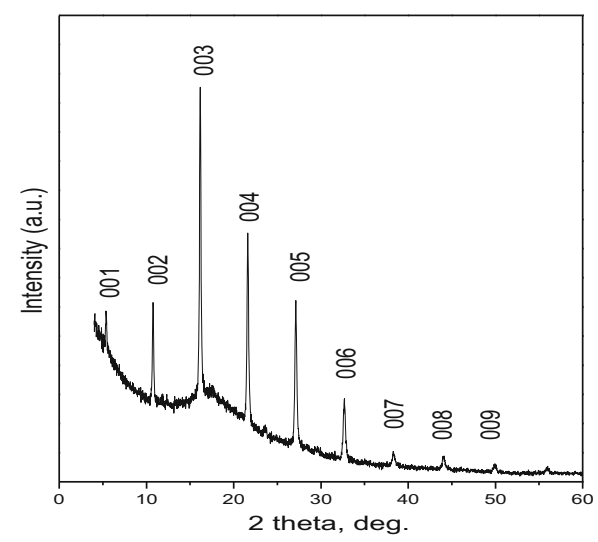

Fig. 6. X-ray diffraction pattern of the conductive sensing layer of the $\mathrm{BL}$ thermistor: polycarbonate/ $\alpha^{\prime}-(\mathrm{BEDT}-\mathrm{TTF})_{2} \mathrm{I}_{\mathrm{x}} \mathrm{Br}_{3-\mathrm{x}}$

\subsection{Integration of BL Thermistor into Textile}

To highlight the formation of the above described crack-like defects, we used a new approach to engineering of the polyester temperature sensing e-textile. We suggest that a rigid flat unit at the polyester textile to which the thermistor has to be attached may be prepared by locally melting of a small part of the textile being sandwiched between two plates. Under local melting the small part of the polyester textile has to lose its textile-like texture while becoming film-like. Due to such procedure, the melted part must become much more rigid as compared to the rest of the textile. 


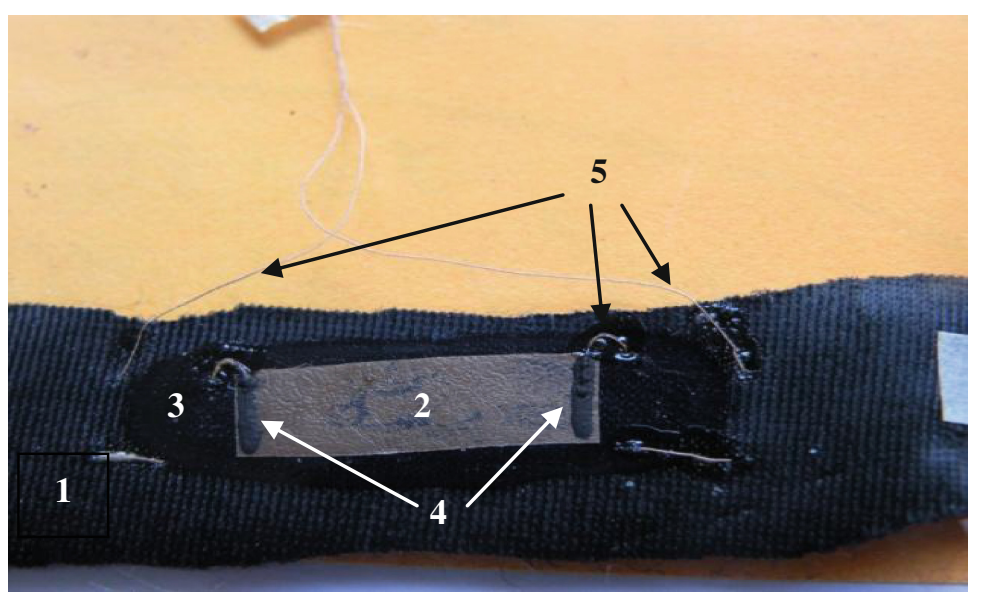

Fig. 7. Photo image of the new prototype of the temperature sensing textile: 1-polyester textile; 2- BL thermistor attached to the flat support (3) which was fabricated by textile melting; 4electrical graphite contacts and 5- wire-based electrical contacts

In order to form the rigid support for the BL thermistors, the part of the textile was sandwiched between two glass slides and heated up to the textile melting point $\left(\cong 250{ }^{\circ} \mathrm{C}\right)$ using soldering iron; the textile plastification process was visually controlled. This procedure allowed us to prepare a smoother sensor support as compared to that fabricating by textile impregnation. The BL thermistor was attached to the rigid polyester-based support using the glue which was unable to destroy the polycarbonate layer of the polycabonate/ $\alpha$ '-(BEDT-TTF $)_{2} \mathrm{I}_{\mathrm{x}} \mathrm{Br}_{3-\mathrm{x}}$ film.

At the final stage of the prototype fabrication, the electrical contacts were attached with graphite paste to the conductive temperature sensing layer of the thermistor (Fig. 7). In fact, the fabricated sensor can be easily interfaced with an embedded system, e.g. a wireless sensor node [28].

The surface of the temperature sensing layer of the BL thermistor was investigated using SEM. Figure 8 shows the sensing layer of the BL thermistor is attached to the rigid melted part of the polyester textile has no imperfectness which can be found in Fig. 4.

\subsection{Temperature Testing of the E-textile Prototype}

We test the e-textile prototype by connecting the sample to a wireless sensor node 'WaspMote'. In particular, we connect the sensor to the Analogue-to-Digital Converter (ADC) located in the Micro Controller Unit (MCU) of the sensor node. This simple testbed is prepared to ensure the proof of concept validation and performing the experiments associated with sensing.

The developed prototype was characterized as a temperature sensor in the temperature range from 23 to $50{ }^{\circ} \mathrm{C}$. Figure 9 shows that the prototype electrical resistance almost linearly depends on temperature. As the resistance of the prototype decreases 


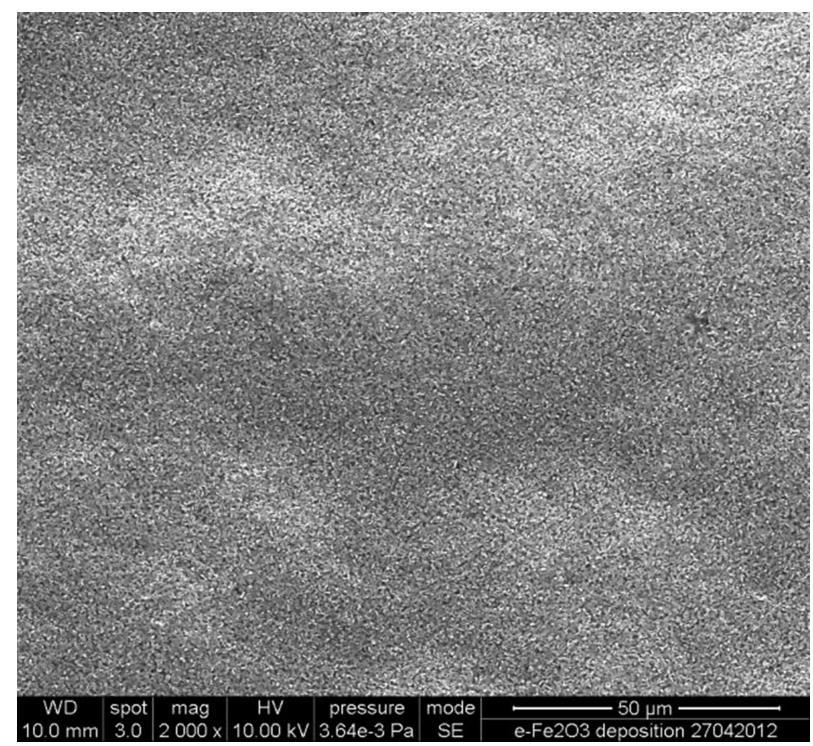

Fig. 8. SEM image of the surface of the BL thermistor attached to the rigid film-like platform which was prepared using molding process.

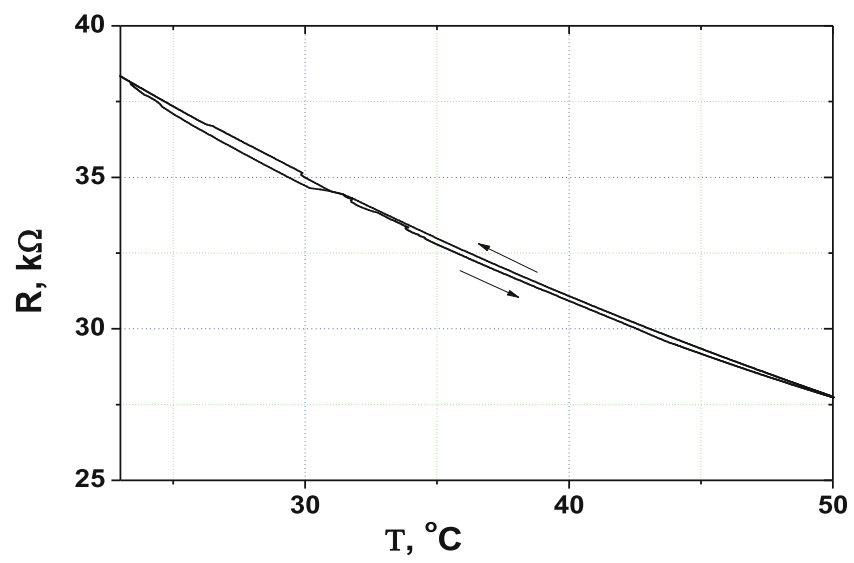

Fig. 9. Resistance temperature dependence of the e-textile equipped with lightweight $\mathrm{BL}$ termistor: polycarbonate/ $\alpha$ '-(BEDT-TTF) $)_{2} \mathrm{I}_{\mathrm{x}} \mathrm{Br}_{3-\mathrm{x}}$

from $38 \mathrm{k} \Omega$ at $23{ }^{\circ} \mathrm{C}$ to $27.6 \mathrm{k} \Omega$ at $50{ }^{\circ} \mathrm{C}$, the sensitivity of the fabricated sample of the e-textile to temperature changes is $0.4 \mathrm{k} \Omega /$ degree. Therefore, the developed BL film is capable of controlling very small temperature changes (0.01-0.005 degree).

The prototype of the temperature sensing e-fabric was additionally subjected to several heating cycles from room temperature up to $60{ }^{\circ} \mathrm{C}$ (Fig. 10).

The data presented in Fig. 10 demonstrate that the electrical response of the fabricated prototype to temperature is reversible, repeatable and stable in time. The 


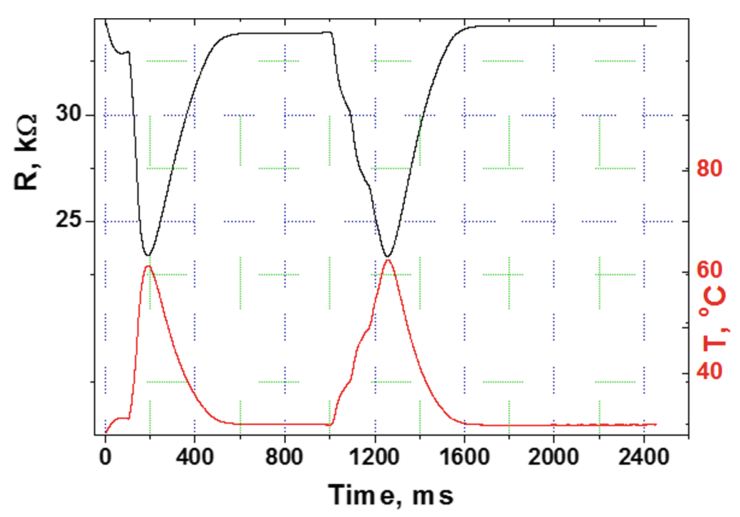

Fig. 10. Electrical response of the developed prototype of the e-textile to temperature cycles.

sensitivity of the conductive fabric to the temperature in the range of the human body temperatures is $-1.2 \% / \mathrm{deg}$; this well corresponds to the previously reported data [27]. This result shows that the developed organic BL film-like thermistor may successfully be used as temperature sensing components in smart wearable fabrics making it possible to measure very small temperature changes in the body temperature range. Additionally, it should be mentioned that biocompatibility testing of the thermistor is in progress.

\section{Future Work}

In our future work we plan a seamless integration of the developed sensing technology, as well as a processing unit and a wireless communication chip, in textile. To realize this idea into practice we are going to adopt the integration techniques developed in the EU Projects, e.g. PASTA (www.pasta-project.eu), Place-it (www.place-it-project.eu).

The integration of the developed sensing technology in textile has a high potential towards a number of monitoring application: environmental sensing (using the temperature and humidity sensors enables one to infer about the fire status), rehabilitation (ECG, EMG, temperature sensors). The application of a wireless technology makes the e-textile an autonomous monitoring system which has high potential in the forthcoming era of the internet of things [29, 30]. This activity requires further research, e.g. in communications [31] and power management [32], to guarantee autonomous operation of the system.

\section{Conclusion}

The BL thermistor: polycarbonate/ $\alpha$ '-(BEDT-TTF $)_{2} \mathrm{I}_{\mathrm{x}} \mathrm{Br}_{3-\mathrm{x}}$ was fabricated and the texture, structure of its conductive sensing layer were investigated using SEM and $\mathrm{X}$-ray analyses. The resistance temperature dependence of the thermistor was also measured. It was showed that all the investigated properties are in good agreement with 
the earlier reported data being indicative of the good reproducibly of the low cost synthetic procedure developed for the lightweight BL thermistor preparation.

A new approach to integrating the BL thermistor into textile was developed: the thermistor was attached to the smooth film-like rigid support prepared directly at the polyester textile by its local melting. It was found that the melted part of the textile is much more rigid as compared to the rest of the fabric.

The fabricated prototype was characterized as a temperature sensor in the temperature range from 23 to $50{ }^{\circ} \mathrm{C}$. The temperature test revealed that the electrical response of the fabricated e-textile to temperature is reversible, repeatable and stable in time. The developed textiles are capable of controlling very small temperature changes with accuracy of $0.005{ }^{\circ} \mathrm{C}$, which is significantly better than that reported for commonly used thermistors; for example, the measurement accuracy of a Pt-1000 detector is $0.01{ }^{\circ} \mathrm{C}$. This kind of e-textile may find a number of applications in biomedical monitoring technologies usually reserved for lightweight highly sensitive temperature sensors with electrical detection principle.

Acknowledgments. The authors acknowledge the financial support from Instituto de Salud Carlos III, through "Acciones CIBER." The Networking Research Center on Bioengineering, Biomaterials and Nanomedicine (CIBER-BBN), an initiative funded by theVINational R\&D\&I Plan 2008-2011, Iniciativa Ingenio 2010, Consolider Program, CIBER Actions and financed by the Instituto de Salud Carlos IIIwith assistance from the European Regional Development Fund. The authors also appreciate the financial support through the projects: BE-WELL (CTQ201340480-R) granted by DGI (Spain), and GenCat (2014-SGR-17) financed by DGR (Catalunya), the European Commission's Seventh Framework Programme for Research under contracts FP7-OCEAN-2013-614155, the Ministry of Education and Science of Russian Federation Grant RFMEFI57714X0133.

\section{References}

1. Brunelli, D., Minakov, I., Passerone, R., Rossi, M.: Smart monitoring for sustainable and energy-efficient buildings: a case study. In: IEEE Workshop on Environmental, Energy and Structural Monitoring Systems (EESMS), pp. 186-191. IEEE Press (2015)

2. Wannenburg, J., Malekian, R.: Body sensor network for mobile health monitoring, a diagnosis and anticipating system. J. IEEE Sens. (2015). doi:10.1109/JSEN.2015.2464773

3. Somov, A., Baranov, A., Spirjakin, D.: A wireless sensor-actuator system for hazardous gases detection and control. J. Sens. Actuators A: Phys. 210, 157-164 (2014)

4. Kalinauckas, A.: Wearable technology. Mag. Eng. Technol. 10, 36-43 (2015)

5. Axisa, F., Schmitt, P.M., Gehin, C., Delhomme, G., McAdams, E., Dittmar, A.: Flexible technologies and smart clothing for citizen medicine, home healthcare, and disease prevention. IEEE Trans. Inf. Technol. Biomed. 9, 325-336 (2005)

6. Lorussi, F., Rocchia, W., Scilingo, E.P., Tognetti, A., De Rossi, D.: Wearable, redundant fabric-based sensor arrays for reconstruction of body segment posture. IEEE Sens. J. 4, 807818 (2004)

7. Lumelsky, V.J., Shur, M.S., Wagner, S.: Sensitive skin. IEEE Sens. J. 1, 41-51 (2001)

8. Ajmera, N., Priya Dash S., Meena, Ch.R.: Smart Textile. http://www.fibre2fashion.com 
9. Stoppa, M., Chiolerio, A.: Sensors wearable electronics and smart textiles: a critical review. Sensors 14, 11957-11992 (2014)

10. Locher, I., Kirstein, T., Troster, G.: Conductive Textiles. http://www.wearable.ethz.ch/ research/groups/textiles/ConductiveTextiles/ambience05_locher.pdf

11. United Nations, New York, Department of Economic and Social Affairs Population Division: World Population Ageing (2013). http://www.un.org/en/development/desa/ population/publications/pdf/ageing/WorldPopulationAgeing2013.pdf

12. Linz, T., Gourmelon, L., Langereis, G.: Contactless EMG sensors embroidered onto textile. In: Leonhardt, S., Falck, T., Mähönen, P. (eds.) BSN 2007, vol. 13, pp. 29-34. Springer, Heidelberg (2007)

13. Löfhede, J., Seoane, F.: Thordstein: soft textile electrodes for EEG monitoring. In: 10th IEEE International Conference on Information Technology and Applications in Biomedicine (ITAB), pp. 1-4. IEEE Press, New York (2010)

14. Löfhede, J., Seoane, F., Thordstein, M.: Textile electrodes for EEG recording - a pilot study. Sensors 12, 16907-16919 (2012)

15. Mittal, K.L.: Preface in Metallized Plastics 7: Fundamental and Applied Aspects, Ed. K.L. Mittal, VSP BV, vii (2001)

16. Waltman, R.J., Bargon, J.: Electrically conducting polymers: a review of the electropolymerization reaction, of the effects of chemical structure on polymer film properties, and of applications towards technology. Can. J. Chem. 64(1), 76-95 (1986)

17. Pimanpang, P.-I., Wang, S., Wang, G.-C., Lu, T.-M.: Self-assembled monolayer growth on chemically modified polymer surfaces. Appl. Surf. Sci. 253, 3532-3540 (2006)

18. Joo, S., Baldwin D.F., Adhesion mechanisms of nanoparticle silver to substrate materials: identification. Nanotechnology, 21, 0552048 (12 p) (2010)

19. Cardoso, M.J.R., Lima, M., Lenz, D.M.: Polyaniline synthesized with functionalized sulfonic acids for blends manufacture. Mater. Res. 10(4), 425-429 (2007)

20. Laukhina, E., Pfattner, R., Ferreras, L.R., Galli, S., Mas-Torrent, M., Masciocchi, N., Laukhin, V., Rovira, C., Veciana, J.: Ultrasensitive piezoresistive all-organic flexible thin films. Adv. Mater. 22, 977-981 (2010)

21. Laukhina, E., Tkacheva, V., Khasanov, S., Zorina, L., Gomez-Segura, J., Perezdel Pino, A., Veciana, J., Laukhin, V., Rovira, C.: Linked crystallites in the conducting topmost layer of polymer bilayer films controlled by temperature: from micro- to nanocrystallites. ChemPhysChem 7, 920-923 (2006)

22. Jerome, D.: Organic conductors: from charge density wave TTF - TCNQ to superconducting (TMTSF)2PF6. Chem. Rev. 104, 5565-5592 (2004)

23. Shibaeva, R.P., Yagubskii, E.B.: Molecular conductors and Superconductors based on Trihalides of BEDT-TTF and some of its analogues. Chem. Rev. 104, 5347-5378 (2004)

24. Saito G.: chap. 10. In: Organic Molecular Solids W. Jones (ed.), CRC, Boca Raton (1997)

25. Ferreras, L., Pfattner, R., Mas-Torrent, M., Laukhina, E., Lopez, L., Laukhin, V., Rovira, C., Veciana, J.: Highly piezoresistive textiles based on a soft conducting charge transfer salt. J. Mater. Chem. 21, 637-641 (2011)

26. PT-100 Series Platinum RTDs. http://www.lakeshore.com/Documents/LSTC_Platinum_1. pdf

27. Laukhina, E., Laukhin, V., Lebedev, V., Rovira, C., Veciana, J.: Conductive fabric responding to extremely small temperature changes. Procedia Eng. 87, 144-147 (2014). 28th European Conference on Solid-State Transducers

28. Somov, A., Lebedev, V., Baranov, A., Laukhina, E., Laukhin, V., Passerone, R., Rovira, C., Veciana, J.: Wireless sensor node with ultrasensitive film sensors for emergency applications. Procedia Eng. 87, 520-523 (2014). 28th European Conference on Solid-State Transducers 
29. Kelaidonis, D., Somov, A., Foteinos, V., Poulios, G., Stavroulaki, V., Vlacheas, P., Demestichas, P., Baranov, A., Biswas, A.R., Giaffreda, R.: Virtualization and cognitive management of real world objects in the internet of things. In: IEEE International Conference on Green Computing and Communications (GreenCom), pp. 187-194. IEEE Press (2012)

30. Miorandi, D., Sicari, S., De Pellegrini, F., Chlamtac, I.: Internet of things: vision, applications and research challenges. J. Ad Hoc Netw. 10, 1497-1516 (2012)

31. Baccour, N., Koubaa, A., Mottola, L., Zuniga, M., Youssef, H., Boano, C., Alves, M.: Radio link quality estimation in wireless sensor networks: a survey. ACM Trans. Sen. Netw. 8, 34 (2012)

32. Somov, A., Baranov, A., Spirjakin, D., Passerone, R.: Circuit design and power consumption analysis of wireless gas sensor nodes: one-sensor versus two-sensor approach. IEEE Sens. J. 14, 2056-2063 (2014) 